\title{
RESEARCH ON ENERGY CONSUMPTION OPTIMIZATION ROUTING PROTOCOL IN DATA TRANSMISSION PROCESS OF WIRELESS SENSOR NETWORK
}

\author{
Chengqiong Ye ${ }^{1}$, Wenyu Shi ${ }^{1}$, Rui Zhang1* \\ ${ }^{1}$ Institute of Information Engineering, Anhui Xinhua University, \\ Hefei, Anhui 230088, China \\ Email: $\underline{\text { nzre78u@126.com }}$
}

\begin{abstract}
In data transmission in a wireless sensor network (WSN), a lot of energy will be consumed, but the energy of WSN is limited. The energy consumption of WSN is optimized to ensure the performance of WSN. This paper first introduced WSN and routing protocol, took Low Energy Adaptive Clustering Hierarchy (LEACH) protocol as the subject, analyzed its workflow and problems, and optimized it with the particle swarm optimization (PSO) algorithm to obtain the PSO-LEACH protocol. The experimental results demonstrated that the Fist Node Die (FND) of the PSO-LEACH protocol appeared in the 946th round and Half Node Die (HND) appeared in the 971st round, both slower than the LEACH protocol, indicating that the PSO-LEACH protocol had a longer life cycle; in the same number of rounds, the energy consumption of the PSO-LEACH protocol was always lower than the LEACH protocol, and the time when all energy was consumed was later than the LEACH protocol; when the energy consumption was the same, the PSO-LEACH protocol received more data than the LEACH protocol. The experimental results show that the improved LEACH protocol can optimize the energy consumption of WSN, which is conducive to energy saving and high efficiency of WSN and can be popularized and applied in practice.
\end{abstract}

Keywords: Wireless Sensor Network, Routing Protocol, Energy Consumption Optimization, Low Energy Adaptive Clustering Hierarchy Protocol, Network Lifetime.

\section{Introduction}

Wireless sensor network (WSN) is composed of many sensor nodes [1], which has the ability of realtime collection, processing, and transmission for the data in the monitoring area. It has the advantages of low cost, small size, and simple deployment. In data processing, it has the advantages of high precision, fewer errors, and remote monitoring. WSN has a very wide application in fields such as environmental monitoring [2], military investigations [3], agricultural management [4], medical health [5], and smart home. The power of WSN is generally supplied by non-rechargeable batteries [6], but in the actual use scenarios, it is usually randomly arranged in the inaccessible environment without continuous energy supply, and the staff can not timely replace the battery. Therefore, the survival time of WSN is greatly limited. For WSN, a very important problem is efficiently utilizing limited energy [7] to realize energy optimization and extend network lifetime [8]. Zhou et al. [9] proposed a multi-request collaborative integration mechanism for WSN, which simplified the selection of WSN into the multi- objective and multi-constraint optimization problem, and solved it with the heuristic algorithm. The experiments found that the method improved the shareability of WSN and significantly reduced the energy consumption of the network. Cao et al. [10] improved the stable election protocol (SEP), which reduced the energy consumption of nodes through the indirect transmission of intermediate nodes, found the best route through the greedy routing algorithm, and found that the improved SEP protocol could balance energy consumption better and prolong network lifetime. Arya et al. [11] analyzed the Ant Colony Optimization (ACO) based routing protocol and compared it with protocols based on gradient and energy-sensing. The results showed that the ACO improved protocol had a better performance. Gupta et al. [12] pointed out that the energy consumption of WSN was related to the size of data packets and the distance of communication and proposed a method to optimize the size of packets to prolong the lifetime of nodes. In this paper, the low energy adaptive clustering hierarchy (LEACH) protocol was optimized by the particle swarm optimization (PSO) algorithm to achieve the balance and optimization of energy consumption, 
which makes some contributions to the energy optimization of WSN.

\section{Wireless Sensor Network}

\section{Overview of wireless sensor network}

In WSN, a large number of nodes are randomly distributed to collect the corresponding information, the information is converted into data and transferred from the common node to the sink node, and the task sensor node that is the processing terminal displays or processes the data transmitted by the node.

The main purpose of WSN is to obtain data information. It has a large scale and wide coverage; therefore, the composition of the network is complex. A large-scale network is also conducive to obtaining more information. Each node has equal status and coordinates to process information.

Due to the characteristic of the multi-disciplinary combination of WSN, its key technology involves many aspects, including (1) routing technology: due to the particularity of WSN, its energy consumption is very critical, and the current research on routing technology is mainly to extend the network life through energy consumption optimization; (2) node positioning [13]: only through the positioning of nodes, can we understand the position that sends the information; (3) data fusion [14]: due to the high density of nodes in WSN, there may be similar data collected by adjacent nodes, causing a large number of duplicate data after sent to sink nodes; therefore, data fusion is needed to ensure the simplification of information; (4) information security $[15,16]$ : WSN uses wireless data transmission; when malicious attacks happen, it will lead to information leakage and a huge loss. Therefore, the information security of WSN should be paid attention to.

\section{Routing protocol}

Routing protocols can be divided into two types. One is the plane routing protocol, in which nodes are self-organized by multiple hops. It is more suitable for small-scale WSN. Different nodes have the same role and status in this kind of protocol and complete data collection together, including flooding protocol, Sensor Protocols for Information via Negotiation (SPIN), directed diffusion protocol, etc. The other is the hierarchical routing protocol, which divides the network into different clusters. Each cluster is composed of cluster head $(\mathrm{CH})$ and cluster member (CM). $\mathrm{CH}$ manages $\mathrm{CM}$ and then sends the processed information to the base station (BS). This method can reduce energy consumption and prolong the network lifetime, including LEACH protocol [17], Power-Efficient Gathering in Sensor Information Systems (PEGASIS) protocol [18], etc. Among them, the LEACH protocol is the earliest and most widely studied. Therefore, based on the LEACH protocol, this study analyzed the energy consumption optimization of WSN.

\section{Energy Consumption Optimization By Routing Protocol}

\section{WSN energy consumption model}

Based on the secondary fading model and polycrystalline attenuation model, in the process of transmitting data from node $i$ to node $j$, it is assumed that the transmission distance is $d_{i j}$, and the data size is $k$ bits. The consumed energy is written as:

$$
\begin{gathered}
E_{\text {send }}\left(k, d_{i j}\right)=\left\{\begin{array}{l}
k E_{\text {elec }}+k \varepsilon_{f s} d_{i j}^{2}, d_{i j}<d_{0} \\
k E_{\text {elec }}+k \varepsilon_{m p} d_{i j}^{4}, d_{i j}>d_{0}
\end{array},\right. \\
d_{i j}=\sqrt{\left(x_{i}-x_{j}\right)^{2}+\left(y_{i}-y_{j}\right)^{2}}, \\
d_{0}=\sqrt{\frac{\varepsilon_{f s}}{\varepsilon_{m p}}},
\end{gathered}
$$

where $E_{\text {elec }}$ stands for energy consumption per unit of data processing, $\varepsilon_{f s}$ and $\varepsilon_{m p}$ are power amplification factors, and $d_{0}$ is a threshold. It is assumed that data reaches the sink node after passing $n$ nodes, the energy consumption model in the above process is written as:

$E_{\text {cost }}=\left\{\begin{array}{l}(2 n+1) \times k \times E_{\text {elec }}+k \times \varepsilon_{f s} \times \sum_{m=1}^{n+1} d_{i j}^{2}, d_{i j}<d_{0} \\ (2 n+1) \times k \times E_{\text {elec }}+k \times \varepsilon_{m p} \times \sum_{m=1}^{n+1} d_{i j}^{4}, d_{i j}>d_{0}\end{array}\right.$.

\section{LEACH routing protocol}

In LEACH protocol, the first step is the establishment stage of clusters. The LEACH protocol adopts the method of random threshold in $\mathrm{CH}$ election. Each node randomly gets a value between 0 and 1. If the value is smaller than threshold $T(n)$, then it can be selected as $\mathrm{CH}$. The calculation method of $T(n)$ is:

$$
T(n)=\left\{\begin{array}{l}
\frac{s}{1-s\left(r \bmod \frac{1}{s}\right)}, i f n \in G \\
0, \text { otherwise }
\end{array},\right.
$$

where $s$ stands for the percentage of $\mathrm{CH}$ in all the nodes, $r$ stands for the number of election rounds, $r$ mod - stands for the number of nodes that are selected as $\mathrm{CH}$ in the current loop, $n$ stands for the 
ID of a node, and $G$ stands for the number of nodes that are not selected as $\mathrm{CH}$ in the current loop.

After the $\mathrm{CH}$ election, when the nodes selected as $\mathrm{CH}$ broadcast that they have become $\mathrm{CH}$ to the whole network, $\mathrm{CM}$ will receive the information from $\mathrm{CH}$ and then select the nearest $\mathrm{CH}$ as their $\mathrm{CH}$. When all nodes have clusters, the cluster establishment ends. The stage of data transmission starts. CM sends the collected data to their $\mathrm{CH}$ according to the preset time slot sequence. $\mathrm{CH}$ receives and fuses the information and sends it to the sink node. It circulates until the network stops operation.

\section{LEACH protocol optimized by PSO}

\section{- PSO algorithm}

In LEACH protocol, the selection of $\mathrm{CH}$ is random, making the distance between two $\mathrm{CH}$ very small, i.e., duplicate data will be received, wasting the energy consumed in data transmission. In the cluster with dense nodes, the energy consumed by $\mathrm{CH}$ was much greater than that of $\mathrm{CH}$ in the cluster with sparse nodes, which will make some $\mathrm{CH}$ die prematurely due to energy shortage. This kind of random $\mathrm{CH}$ election will lead to the imbalance of cluster. Therefore, the PSO algorithm is selected in this study for optimization.

It is assumed that in a $D$-dimensional space, the number of particles is $N$, the particle swarm is $X=\left(x_{1}, x_{2}, \cdots, x_{n}\right)$, the position and speed of particle $i$ are $x_{i}=\left(x_{i 1}, x_{i 2}, \cdots, x_{i d}\right)$ and $v_{i}=\left(v_{i 1}, v_{i 2}, \cdots, v_{i d}\right)$, respectively, and the individual optimal and global optimal are $p_{i}=\left(p_{i 1}, p_{i 2}, \cdots, p_{i d}\right)$ and $p_{g}=\left(p_{g 1}, p_{g 2}, \cdots, p_{g d}\right)$, respectively. The particle updates its speed according to $v_{i}(t+1)=w v_{i}(t)+c_{1} r_{1}\left(p_{i d}-x_{i}(t)\right)+c_{2} r_{2}\left(p_{g d}-x_{i}(t)\right) \quad$ and updates its position according to $x_{i}(t+1)=x_{i}(t)+v_{i}(t+1)$, where $t$ stands for the number of iterations, $w$ stands for the inertia factor, $r_{1}$ and $r_{2}$ are random numbers in [0,1], and $c_{1}$ and $c_{2}$ are learning factors, constants between 0 and 2 .

\section{- PSO-LEACH protocol}

It is assumed that WSN has $N$ nodes, and it needs to be divided into $M$ clusters. Firstly, WSN is divided into two parts using the PSO algorithm. The number of nodes in these two regions is the same. The dividing line can be written as: $L=(x, y, \theta)$, where $(x, y)$ is the point coordinates on the dividing line and $\theta$ is the angle between the dividing line and $x$ axis. The fitness function is written as: fitness $=\left(c_{1}-f_{1} N\right)^{2}+\left(c_{2}-f_{2} N\right)^{2}, \quad f_{i}=\frac{M_{i}}{M}, i=1,2$, where $M_{i}$ refers to the number of $\mathrm{CH}$ that are expected to be reserved through division.

The process of the PSO-LEACH protocol can be described as follows. Firstly, all nodes in the network send their state information to BS, and BS clusters through the PSO algorithm according to the information. $K$ particles are defined. The whole WSN is divided into $K \times 2$ subregions using the formula mentioned above. Then the fitness functions of the subregions are calculated. According to $p_{i d}$ and $p_{g d}$, based on the position and speed updating formulas of the PSO algorithm, the positions and speeds of $x, y$, and $\theta$. Through the continuous updating of particles, the whole WSN has been divided into two regions with the same size after the maximum times of iterations is reached. Then, the sub-regions are further divided according to the same method until the whole WSN is divided into $M$ clusters of the same size. After that, the $\mathrm{CH}$ election started. To optimize energy consumption, the node with larger residual energy should be selected as $\mathrm{CH}$. An energy threshold is set as $\mathrm{E}$. If the residual energy of the node is larger than $\mathrm{E}$, then the node is named a high-energy node; the other nodes are named ordinary nodes. It is assumed that the proportion of high-energy nodes in all the nodes is $q$, the part of the energy that is higher than the ordinary node is $\vartheta$, then the probabilities that the two kinds of nodes are elected as $\mathrm{CH}$ are written as:

$$
\begin{aligned}
& \rho_{\text {normal }}=\frac{s}{1+\vartheta \times q} \\
& \rho_{\text {high }}=\frac{s}{1+\vartheta \times q} \times(1+\vartheta),
\end{aligned}
$$

where $s$ stands for the percentage of $\mathrm{CH}$ to all the nodes. The calculation formula of threshold $T(n)$ in the LEACH protocol is updated using the calculated probabilities:

$$
\begin{aligned}
& T\left(n_{\text {normal }}\right)=\left\{\begin{array}{l}
\frac{\rho_{\text {normal }}}{1-\rho_{\text {normal }}\left(r \bmod \frac{1}{\rho_{\text {normal }}}\right)}, \text { if } n_{\text {normal }} \in G^{\prime}, \\
0, \text { otherwise }
\end{array}\right. \\
& T\left(n_{\text {high }}\right)=\left\{\begin{array}{l}
\frac{\rho_{\text {high }}}{1-\rho_{\text {high }}\left(r \bmod \frac{1}{\rho_{\text {high }}}\right)}, i f n_{\text {high }} \in G^{\prime \prime} \\
0, \text { otherwise }
\end{array}\right.
\end{aligned}
$$

where $G^{\prime}$ and $G^{\prime \prime}$ are the number of ordinary nodes and high-energy nodes that are not elected as $\mathrm{CH}$ in the current loop. 


\section{Simulation Analysis}

\section{Experimental Environment}

The experiment was carried out in the MATLAB environment. The network size was $100 \times 100 \mathrm{~m}$ (Figure 1). There were 100 nodes in total. The coordinate of BS was (50,50). The initial energy of the node was $0.8 \mathrm{~J}$. The required parameters are shown in Table 1.

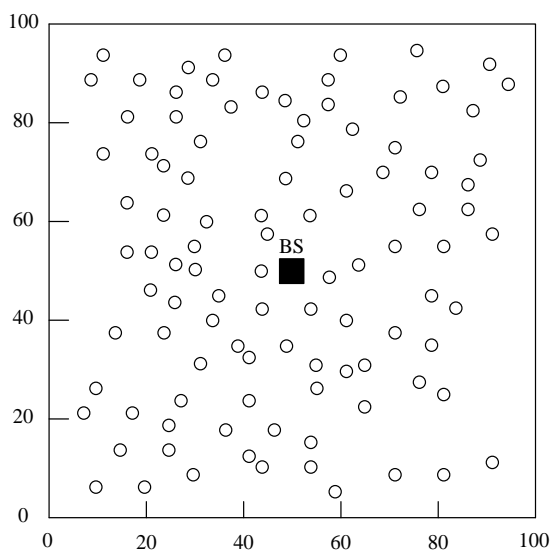

Figure 1: Simulation Network

Table 1. Experimental Parameters

\begin{tabular}{|l|l|}
\hline Parameter & Numerical value \\
\hline$E_{\text {elec }}$ & $50 \mathrm{~nJ} / \mathrm{bit}$ \\
\hline$\varepsilon_{f x}$ & $10 \mathrm{pJ} / \mathrm{bit} / \mathrm{m}^{2}$ \\
\hline$\varepsilon_{m p}$ & $0.0013 \mathrm{pJ} / \mathrm{bit} / \mathrm{m}^{4}$ \\
\hline$d_{0}$ & $87.7 \mathrm{~m}$ \\
\hline $\begin{array}{l}\text { Length of the data } \\
\text { package }\end{array}$ & $4000 \mathrm{bits}$ \\
\hline $\begin{array}{l}\text { Maximum number of } \\
\text { simulation rounds }\end{array}$ & 1000 rounds \\
\hline
\end{tabular}

\section{Result Analysis}

The LEACH protocol was compared with the PSOLEACH protocol. First of all, the first node die (FND) and half node die (HND) of the two protocols were compared. The later the two indicators appeared, the longer the working life of the network was. The results are shown in Figure 2.

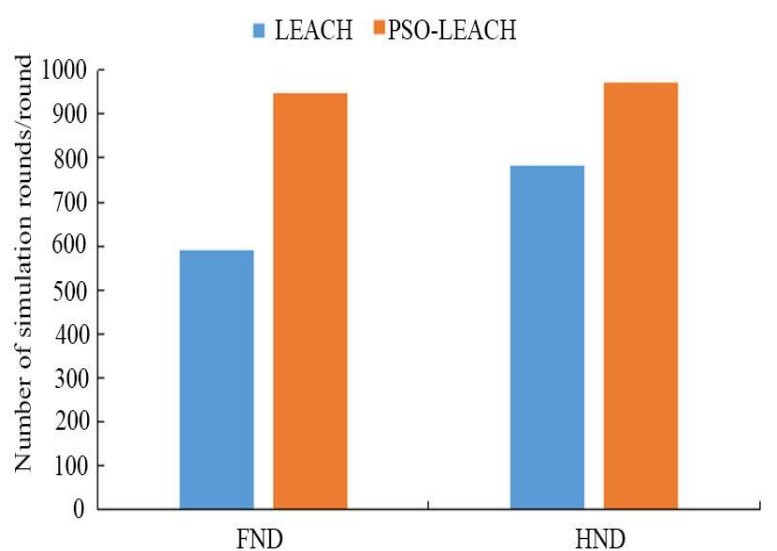

Figure 2: Comparison of Network Working Life

It was seen from Figure 2 that the FND and HND of the LEACH protocol appeared after 589 round and 782 rounds, respectively, the FND and HND of the PSO-LEACH protocol appeared after 946 rounds and 971 rounds, respectively. The appearance of the FND and HND of the PSO-LEACH protocol was delayed; the appearance time of FND was $60.61 \%$ longer than the LEACH protocol, and the appearance time of HND was $24.17 \%$ longer than the LEACH protocol. Failure of half nodes means network failure. It was found that the PSO-LEACH protocol greatly extended the working life of WSN. Also, the PSO-LEACH protocol only experienced 25 rounds from FND to HND, which was far less than 193 rounds of the LEACH protocol. Through the specific analysis, it was found that the random $\mathrm{CH}$ election mode of the LEACH protocol led to uneven distribution of $\mathrm{CH}$ in the early stage of network operation; as a result, some nodes used up the energy too early and died, but some nodes did not use up the energy as they failed to select the effective $\mathrm{CH}$ and died after many rounds. Therefore, the LEACH protocol experienced more rounds from FND to HND, which was also a manifestation of uneven energy consumption. In the PSO-LEACH protocol, the election of $\mathrm{CH}$ was improved by the PSO algorithm, which made the clusters of WSN more balanced, and the energy consumption of each round was evenly distributed to every node; therefore, there were fewer rounds from FND to HND.

The comparison of the remaining energy of the whole network is shown in Figure 3. 


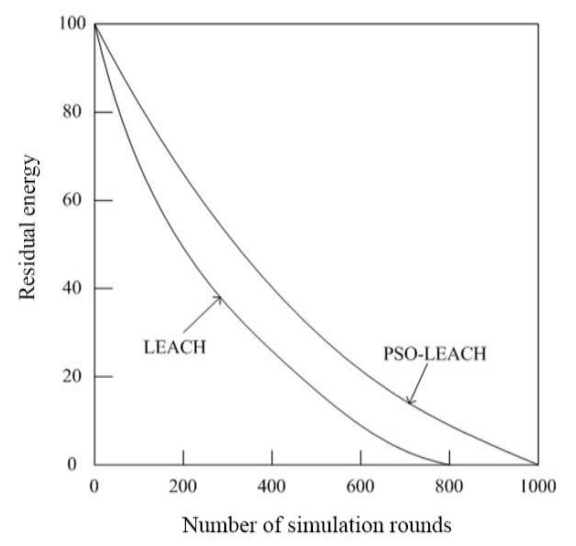

Figure 3: Comparison of Residual Network Energy

It was seen from Figure 3 that the residual energy of the LEACH protocol was always lower than that of the PSO-LEACH algorithm when the number of rounds was the same; when the number of rounds was about 800, the energy of the LEACH protocol has been exhausted, while that of the PSO-LEACH algorithm was exhausted after 1000 rounds. It showed that the PSO-LEACH algorithm was more balanced in energy consumption.

The change in the amount of data received by the sink node under different energy consumption conditions was compared, and the results are shown in Figure 4.

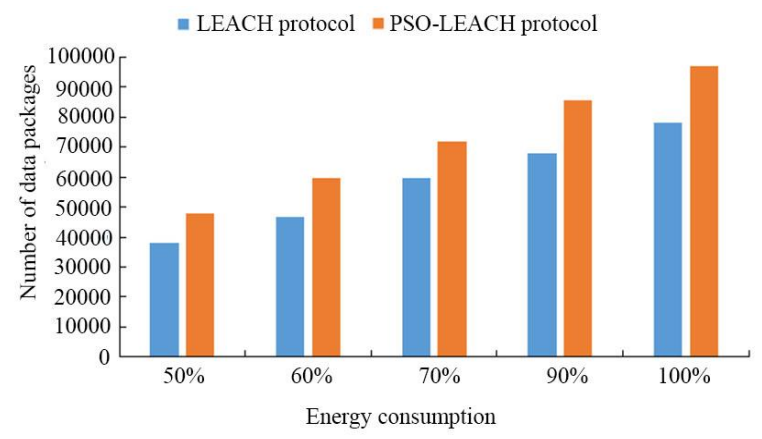

Figure 4: Comparison of Received Data Volume

It was seen from Figure 4 that the LEACH protocol received 38000 data packages, and the PSOLEACH protocol received 48000 data packages (26.32\% more than the LEACH protocol) when the energy consumption of WSN was $50 \%$. With the increase of energy consumption, the number of received data package also increased. When the energy consumption reached $100 \%$, the LEACH protocol received 78000 data packages, while the PSO-LEACH protocol received 97000 data packages, which was $24.36 \%$ more than the LEACH protocol. It indicated that the PSO-LEACH protocol was more energy-saving and efficient and could transmit more data with better performance under the same energy consumption.

\section{Conclusions}

In this study, taking the LEACH protocol as the subject, aiming at the problem of the random election of $\mathrm{CH}$, the protocol was improved by the PSO algorithm to obtain the PSO-LEACH protocol, and the experiment was carried out to compare the PSOLEACH protocol with the LEACH protocol. The results showed that:

(1) FND and HDN appeared later in the PSOLEACH protocol, and the network life cycle was longer;

(2) under the same number of rounds, the PSOLEACH protocol consumed less energy than the LEACH protocol;

(3) the PSO-LEACH protocol used up the energy later than the LEACH protocol;

(4) under the same energy consumption, the sink node in the PSO-LEACH protocol received more data.

The simulation results show that the PSO-LEACH protocol has a significant effect on energy consumption optimization, which can balance the energy consumption of nodes to realize the network's energy saving, prolong the working life of the network, and maintain a high working efficiency. The PSO-LEACH protocol can be applied in WSN in reality.

\section{References}

[1] Ji D, Wang X. "Research on Remote Monitoring Protocol of Power System Based on Wireless Sensor Network," IOP Conference Series: Materials Science and Engineering, 2018, 452(3):032123 (4pp).

[2] Liu J, Li L J, Liu Y X. "An Environment Monitoring System Based on Wireless Sensor Networks," Advanced Materials Research, 2015, 765767:2109-2112.

[3] Ghosh K, Neogy S, Das P K, Mehta M. "Intrusion Detection at International Borders and Large Military Barracks with Multi-sink Wireless Sensor Networks: An Energy Efficient Solution," Wireless Personal Communications, 2017, 98(2):1-19.

[4] Ojha T, Misra S, Raghuwanshi N S. "Wireless sensor networks for agriculture: The state-of-theart in practice and future challenges," Computers \& Electronics in Agriculture, 2015, 118:66-84.

[5] Hassaballah H J, Fayadh R A. "Implementation of wireless sensor network for medical applications," IOP Conference Series: Materials Science and Engineering, 2020, 745(1):012089 (10pp).

[6] Luo J, Hu J, Wu D, Li R F. “Opportunistic Routing Algorithm for Relay Node Selection in Wireless Sensor Networks," IEEE Transactions on Industrial Informatics, 2017, 11(1):112-121. 
[7] Vijayabaskar K, Kumanan T. "Link Consistency and Energy Efficiency for Improving Throughput in Wireless Sensor Network," IOP Conference Series: Materials Science and Engineering, 2020, 925(1):012073 (11pp).

[8] Kuila P, Jana P K. "Energy Efficient Load-Balanced Clustering Algorithm for Wireless Sensor Networks," Procedia Technology, 2012, 6(1):771777.

[9] Zhou Z, Xu J, Zhang Z, Lei F, Fang W. "Energyefficient optimization for concurrent compositions of WSN services," IEEE Access, 2018, PP(99):1-1.

[10] Cao Y, Zhang L. "Energy optimization protocol of heterogeneous WSN based on node energy," 2018:495-499.

[11] Arya R, Sharma S C. "Optimization approach for energy minimization and bandwidth estimation of WSN for data centric protocols," International Journal of Systems Assurance Engineering and Management, 2018, 9, 2-11.

[12] Gupta A L, Shekokar N. "A novel approach to improve network lifetime in WSN by energy efficient packet optimization," 2016:117-122.

[13] Silva H D, Afonso J A, Rocha L A. "Body Attenuation and Path Loss Exponent Estimation for RSS-Based Positioning in WSN," Wireless Personal Communications, 2016, 94:1-23.
[14] Xiao L, Jian Y. "Wireless Sensor Network Data Fusion Model Based on Compressed Sensing Theory," Journal of Computational and Theoretical Nanoscience, 2016, 13(12):95159520.

[15] Zhang Y, Liu W, Lou W, Fang Y. "Locationbased compromise-tolerant security mechanisms for wireless sensor networks," IEEE Journal on Selected Areas in Communications, 2015, 24(2):247-260.

[16] Song H, Zhou J. "Design and Implementation of Wireless Sensor Network Re-encryption Protocol Based on Key Distribution," Journal of Physics: Conference Series, 2019, 1345(5):052037 (8pp).

[17] Sohn I, Lee J H, Lee S H. "Low-Energy Adaptive Clustering Hierarchy Using Affinity Propagation for Wireless Sensor Networks," IEEE Communications Letters, 2016, 20(3):558-561.

[18] Lindsey S, Raghavendra. "PEGASIS: Powerefficient gathering in sensor information systems," Aerospace Conference Proceedings, 2003. 